\title{
Tropical Soil Metagenome Library Reveals Complex Microbial Assemblage
}

\author{
Kok-Gan Chan ${ }^{1 \#}$ and Zahidah Ismail ${ }^{1}$
}

${ }^{1}$ Division of Genetics and Molecular Biology, Institute of Biological Sciences, Faculty of Science, University of Malaya, Kuala Lumpur, Malaysia

\# Author to whom correspondence should be addressed; E-Mail: kokgan@um.edu.my; Tel.: +603-7967-5162; Fax: +603-7967-4509. 


\section{$2 \underline{\text { Abstract }}$}

3 In this work, we characterized the metagenome of a Malaysian mangrove soil sample via next

4 generation sequencing (NGS). Shotgun NGS data analysis revealed high diversity of microbes

5 from Bacteria and Archaea domains. The metabolic potential of the metagenome was

6 reconstructed using the NGS data and the SEED classification in MEGAN shows abundance of

7 virulence factor genes, implying that mangrove soil is potential reservoirs of pathogens.

8

9 Keywords: Metagenomics, Mangrove, Soil, Proteobacteria 


\section{Introduction}

12 Mangrove forests are usually located at the tropical and subtropical latitudes. They are present at

13 the transition of land and sea which makes them susceptible to tidal change and salinity.

14 Mangrove soils generally comprise soft, muddy and anaerobic sediment with thin top layer of

15 aerobic sediment. They also function as heavy metal $\operatorname{sink}^{1}$ which acts as natural sink and

16 filtration system. Mangrove swamps are the habitat for a diverse variety of fauna especially

17 juvenile fishes, and they also act as breeding and nursery grounds for these aquatic animals.

18 Microorganisms in the mangrove habitat play an important role in maintaining the productivity,

19 conservation and nutrients of this ecosystem. Microorganisms are involved in biogeochemical

20 cycles that supply nutrients to plants and animals ${ }^{2,3}$. Mangroves are rich in organic matters but

21 usually lack phosphorus and nitrogen ${ }^{4-7}$. Their microorganisms' activities are high because they

22 are very efficient in recycling the nutrients contained therein. Microorganisms are directly

23 involved in nitrogen fixation, phosphate solubility, photosynthesis, sulfate reduction and

24 production of other substances.

25 The mangrove environment is highly susceptible to anthropogenic effects such as pollution,

26 deforestation and human activity. These could change the dynamic mangrove ecosystem which in

27 turn affects the mangrove community and disturbs the microorganism community that maintains

28 the productivity and conservation of mangrove.

29 This study aimed to investigate the metagenome of a mangrove soil sample and their ecological

30 role via metabolic reconstruction. We used the Illumina HiSeq 2000 platform to carry out

31 shotgun metagenome next generation sequencing (NGS). This method avoided bias of PCR

32 amplification as in the case of amplicon sequencing and it enabled parallel study on both the 
taxonomic and functional diversities. We hypothesized that the abundance and diversity of microbes and their functional attributes to be similar to those of previous studies ${ }^{8,9}$.

\section{Materials and Methods}

Sampling was done on a soil sample obtained in the east coast of Peninsular Malaysia, namely

Rantau Abang (RA) (N04 ${ }^{\circ} 54.189^{\prime}$ E103 $\left.{ }^{\circ} 22.208^{\prime}\right)$. No specific permissions were required for the

chosen locations and such research activities. Our work also did not involve endangered or protected species. The top 5 to $20 \mathrm{~cm}$ of soil was collected and stored at $-20^{\circ} \mathrm{C}$ until processing. A portion of the soil sample was sent for biochemical analyses of its $\mathrm{pH}$, carbon nitrogen ratio, and

41 contents of phosphorus, sulfur and heavy metals, like arsenic, cadmium, lead and mercury, as 42 described previously ${ }^{10}$.

43 DNA extraction was carried out according to the protocol as described previously ${ }^{11}$ with 44 modifications. Traces of plant materials were removed from the soil prior to extraction. Briefly,

$455 \mathrm{~g}$ of soil was added with $13.5 \mathrm{ml}$ of DNA extraction buffer (Tris-HCl, pH8 100mM; EDTA, pH8

$\left.46100 \mathrm{mM} ; \mathrm{Na}_{2} \mathrm{HPO}_{4}, \mathrm{pH} 7.8100 \mathrm{mM} ; \mathrm{NaCl}, 1.5 \mathrm{M} ; \mathrm{CTAB}, 1 \% \mathrm{w} / \mathrm{v}\right), 100 \mu \mathrm{l}$ of proteinase $\mathrm{K}$

$47(10 \mathrm{mg} / \mu \mathrm{l})$, and $200 \mu \mathrm{l}$ of lysozyme $(10 \mathrm{mg} / \mu \mathrm{l})$. The mixture was incubated horizontally at $37^{\circ} \mathrm{C}$

48 with orbital shaking $(225 \mathrm{rpm})$. After 30min, $0.5 \mathrm{ml}$ of SDS $(20 \% \mathrm{w} / \mathrm{v})$ was added and the mixture

49 further incubated in a $65^{\circ} \mathrm{C}$ water bath for $2 \mathrm{~h}$ with gentle mixing by inverting the tube at $15 \mathrm{~min}$

50 intervals. The supernatant was collected by centrifugation at $6000 \times \mathrm{g}$ for $10 \mathrm{~min}$. The pellet was

51 suspended in $4.5 \mathrm{ml}$ of DNA extraction buffer and $0.5 \mathrm{ml}$ of SDS $(20 \% \mathrm{w} / \mathrm{v})$ and vortexed for $10 \mathrm{~s}$

52 followed by incubation at $65^{\circ} \mathrm{C}$ for $10 \mathrm{~min}$. The supernatant was then collected by centrifugation

53 and pooled with the supernatant collected previously. Equal volume of chloroform:isoamyl

54 alcohol $(24: 1, \mathrm{vol} / \mathrm{vol})$ was added to the pooled supernatant and the mixture was gently mixed by 
55 inversion. The aqueous phase was transferred to a clean, sterile tube after centrifugation at $6000 \times$

$56 g$ for 10min. The chloroform:isoamyl alcohol step was repeated once. For DNA precipitation, 0.6

57 volume of cold isopropanol was added and the resultant mixture incubated at $-20^{\circ} \mathrm{C}$ for $30 \mathrm{~min}$.

58 DNA was collected by centrifugation at $16000 \times g$ for $20 \mathrm{~min}$, followed by washing with $70 \%$

$59(\mathrm{v} / \mathrm{v})$ ethanol and kept in $-20^{\circ} \mathrm{C}$ for $15 \mathrm{~min}$. Ethanol was removed by centrifugation at top speed in

60 a table top centrifuge for 10min and the pellet was air dried aseptically. The DNA pellet was then

61 dissolved in elution buffer (Roche High Pure PCR Product Purification Kit).

62 The soil metagenomic DNA was further purified by gel elution in a $3 \%(\mathrm{w} / \mathrm{v})$ low melting 63 temperature agarose electrophoresis. The metagenomic DNA was mixed with $80 \%(\mathrm{v} / \mathrm{v})$ glycerol 64 and $6 \times$ loading dye and the mixture was then loaded into a well. Electrophoresis was carried out at $15 \mathrm{~V}$ for 16 to $20 \mathrm{~h}$. DNA was excised from the gel with a sterile blade and recovered using the

66 Qiagen Gel Extraction Kit (Venlo, Netherlands). DNA concentration and purity were determined

67 using Qubit and Nanodrop 2000c by Life Technologies, respectively. The purified DNA was then 68 subject to NGS using Illumina HiSeq 2000.

69 For taxonomic analysis, the metagenomic nucleotide sequences obtained were trimmed using 70 CLC Bio Genomic Workbench 5.5.2 (Aarhus, Denmark) at 50-nucleotide length to remove short

71 low quality reads. The trimmed data were then blasted against the NCBI Microbial database

72 (dated 22 Jan 2013) using Blastall 2.2.25 (NCBI) at the expected value of $1 \times 10^{-20}$.

73 For functional gene study, the trimmed nucleotide sequences were assembled using de novo 74 assembly in CLC Bio Genomic Workbench at the minimum contig length of 400 nucleotides.

75 The assembled data were extracted at coverage of $\geq 10 \%$. Gene prediction was performed on the 76 extracted sequences using Prodigal $2.60^{12}$, and each predicted gene was annotated using 77 RAPsearch $2.09^{13,14}$ (against the NCBI NR database (dated 22 January 2013). Data obtained for 
78 both taxonomic and functional distributions were analyzed in MEGAN 4.70.4 ${ }^{15}$. Taxonomic 79 analysis was done according to the percentage identity filter to get the best sequence match. In 80 MEGAN, functional analysis was accomplished with the $\mathrm{SEED}^{16}$ classification.

81 The data of this study are available as NCBI database accession number SRR748204.

\section{$82 \quad \underline{R e s u l t s}$}

83 Biochemical Analyses

84 Table 1 shows the biochemical properties of the Rantau Abang (RA) soil sample. The pH for RA 85 sample was recorded as $\mathrm{pH}$ of 5.1.

$86 \quad$ Metagenomic Library Analysis

87 The RA sample metagenome library shows very high nucleotides sequenced after editing and $88>500,000$ contigs generated at the coverage at approximately 41 times (Table 2).

$89 \quad$ Microbial Taxonomic Distribution

90 A total of $98 \%$ of the reads from RA sample was assigned to the domains level by MEGAN and

91 they excluded the "No hits" reads category. The majority of the assigned reads from RA samples

92 was of the domain Bacteria while the remaining were of the domain Archaea with $78.52 \%$ reads

93 assigned to Bacteria and $21.48 \%$ to Archaea (Figure 1).

94 There were 27 phyla hits from the domain Bacteria for RA metagenome library. The phylum

95 Proteobacteria dominated other phyla in $(43.72 \%)$ in the RA sample. There were 10 phyla with 96 abundance percentages of more than $1 \%$ in the RA sample. In this sample, the phyla detected 97 were Proteobacteria, Acidobacteria (17.68\%), Firmicutes (13.45), Actinobacteria (4.55\%), 
Nitrospirae (4.22\%), Planctomycetes (3.06\%), Chloroflexi (2.88\%), Verrucomicrobia (2.69\%),

99 Spirochaetes (1.70\%), Chlamydiae (1.32\%) and Bacteroidetes (1.31\%) (Figure 2a). In RA sample, unclassified bacteria were clustered as Caldithrix, Haloplasmatales and some phototrophic

101 bacteria.

102 Forty-three classes of bacteria were detected in RA sample (Figure 2b) where the first two most 103 abundant classes were Deltaproteobacteria (19.29\%) and Alphaproteobacteria (16.89\%), followed 104 by Acidobacteria (16.61\%) (Figure 3b). The other minor classes included Clostridia (9.32\%), 105 Gammaproteobacteria (5.50\%), Actinobacteria (4.58\%), Nitrospira (4.24\%), Bacilli (3.24\%), 106 Planctomycetia (3.08\%), Spirochaetia (1.71\%), Betaproteobacteria (1.69\%), Opitutae (1.52\%), 107 Chlamydia (1.33\%), Verrucomicrobiae (1.19\%), Holophagae (1.17\%), Anaerolineae (1.06\%), 108 and Ktedonobacteria (1.02\%).

At the genus level, Acidobacterium of the Acidobacteria phylum was the dominant genus in RA sample. The abundance frequencies of this genus is $10.01 \%$ (Figures $2 \mathrm{c}$ ).

111 Five classes of Proteobacteria namely Alphaproteobacteria, Betaproteobacteria, 112 Deltaproteobacteria, Gammaproteobacteria and Epsilonproteobacteria, were detected in RA 113 sample). Among these, Deltaproteobacteria (43.88\%) was the major class, followed by 114 Alphaproteobacteria (38.43\%), Gammaproteobacteria (12.51\%) Betaproteobacteria (RA 3.85\%) 115 and Epsilonproteobacteria (RA 1.33\%) (Figure 3). The segregation of orders within 116 Deltaproteobacteria (Figure 4) showed Syntrophobacterales was the most abundant order in this 117 soil sample. At the genus level of this order Syntrophobacter was the most abundant genus 118 (Figures 5). 
119 The RA sample showed present of archaea but only phyla Crenarchaeota and Euyarchaeota were

120 detected. In the RA sample, Crenarchaeota (63.78\%) was present at a higher percentage as

121 compared to Euyarchaeota (36.22\%) (Figure 6). A total of eight classes of archaea were detected

122 in both soil samples and among them, Thermoprotei (RA 63.78\%) and Methanomicrobia (RA

$12317.85 \%)$ were the two dominant classes. Other minor archaea classes present in the RA sample

124 were Thermococci (5.48\%), Methanococci (5.35\%), Thermoplasmata (3.52\%), Methanobacteria

125 (2.17\%), Archaeoglobi (1.36\%) and Halobacteria (0.47\%).

126 The taxonomic diversity for the domains Bacteria and Archaea was estimated at the genus level

127 using the Shannon-Weaver diversity index, H', in MEGAN and the H' value RA samples was

$128 \quad 7.765$.

129 Metabolic Functional Analysis via Reconstruction of Metagenome Library

130 The gene anthology was derived from the SEED classifications. Using this approach, the most

131 abundant gene detected in the RA sample was associated with carbohydrate metabolism (12.97\%).

132 The second most abundant genes in the RA sample were associated with protein metabolism $133(9.89 \%)$, virulence $(9.53 \%)$, respiration $(8.39 \%)$ and amino acids and their derivatives $(8.19 \%)$

134 (Figure 7).

\section{Discussion}

136 The application of the NGS enables the study of microbial diversity and function in metagenomes

137 without the need of culturing bacteria thus bypassing the growing of fastidious bacteria, which 138 are often unculturable, on laboratory media. However, this method depends on the reliability of 139 NGS data generated. 
140 Most bacteria detected in the RA metagenomes were either anaerobic or facultative anaerobic.

141 They were predominantly from the domain Bacteria. However, in the RA metagenome, a

142 significant number of bacteria belonging to the domain Archaea were detected and the percentage

143 of archaeal abundance $(21.48 \%)$ is significantly higher than the percentage values reported for

144 other soil metagenomes ${ }^{17}$. Crenarchaeota is known to be present at high sulfur content

145 environment and for its ability to utilize sulfur ${ }^{18}$. The high percentage of this phylum detected in

146 the RA sample is consistent with the high concentration of sulfur in the RA sample.

147 The bacterial diversity detected in the RA sample conforms to the common soil bacteria present

148 in other types of soil in other geographical locations ${ }^{19}$. In our RA sample, Proteobacteria remains

149 the most abundant bacteria and it comprised five different classes. However, the distribution of

150 Proteobacteria classes in this study differs from the distributions of Proteobacteria classes

151 reported for other mangrove habitats ${ }^{8,20}$. In contrast to other mangrove metagenomes reported to

152 date, both the Malaysian mangrove metagenome possessed Deltaproteobacteria as the dominant

153 class of Proteobacteria. Even though Proteobacteria was the dominant phylum in RA

154 metagenome, Acidobacterium of the Acidobacteria phylum was the most abundant genus in our

155 soil sample.

156 The presence of the high frequency of genes associated with carbohydrate metabolism in our soil

157 metagenome analysis is not surprising because these genes are commonly detected in abundance

158 in most studies of soil metagenomes ${ }^{8,21}$. However, the presence of the high frequency of

159 virulence factor genes in our soil metagenomes is unusual because they are not commonly

160 reported $^{8,22}$. This leads to the speculation that mangrove soil is a potential reservoir of pathogenic

161 bacteria but further work is required to verify this finding. 
162 In our RA soil metagenome, antibiotic and toxic compound resistance genes were also detected

163 frequently. Their abundance may be related to the high percentages of Actinobacteria, which are

164 known to produce a myriad of antibacterial compounds, and Deltaproteobacteria, members of

165 which are known to be resistant to heavy metals and to oxidize heavy metals to their benign

166 forms $^{23}$, in our two tropical mangrove soil samples. We also obtained a high hit rate of the stress

167 response gene in our soil metagenomic libraries, suggesting that the tropical mangrove

168 environment is harsh for microorganisms. This is most probably due to polluted marine waters

169 and high salinity and low aeration available in the muddy mangrove soil.

170 The biochemical tests showed considerably high amount of phosphorus and sulfur in our soil

171 sample. However, the gene ontology analysis revealed that the genes associated with the

172 metabolism of compounds containing phosphorus and sulfur were of relatively low abundance,

173 suggesting that these compounds may mainly be involved in redox reactions in electron transport

174 but not in microbial metabolism. This also implies that sulfur and phosphorus compounds exist in

175 stable forms as mangroves are sink for inorganic compounds.

176 Although Actinobacteria and Firmicutes were two of the detected sub-dominant phyla, our

177 analysis showed low frequency of spore producing bacteria such as Bacillus and Streptomyces.

178 This may explain why the genes for sporulation and dormancy were not detected in our soil

179 metagenomic DNA.

\section{Conclusion}

181 This study demonstrated the high level of microbial diversity in mangrove swamps compared to

182 the limited vegetation that is able to survive in this environment. The high abundance of members

183 of Deltaproteobacteria and heavy metal and toxic compound resistance genes indicates that 
184 microorganisms have potential for bioremediation of heavy metals. The differences in the

185 distribution of microorganisms compared with other previous studies on mangrove soils are most

186 likely due to the different geographical locations. To the best of our knowledge, this is the first

187 study of microbial diversity of mangrove soil in Malaysia using the NGS metagenomic approach.

188 More mangrove soil samples collected from different locations in Malaysia are required to be

189 analysed by this approach before a more defined conclusion on the microbiome of Malaysian

190 mangrove soils and its functional genes can be reached. 


\section{Acknowledgements}

193 Kok-Gan Chan thanks the University of Malaya for the High Impact Research Grants 194 (UM.C/625/1/HIR/MOHE/CHAN/01, Grant No. A-000001-50001 and UM-MOHE HIR Grant 195 UM.C/625/1/HIR/MOHE/CHAN/14/1, no. H-50001-A000027). There are no conflicts among 196 authors regarding this research.

197 
198

199

200

201

202

203

204

205

206

207

208

209

210

211

212

213

214

215

\section{$\underline{\text { References }}$}

[1] Tama NFY, Wong YS., Retention and distribution of heavy metals in mangrove soils receiving wastewater. Environ Pollut, 1996, 94(3): 283-291.

[2] Holguin G, Vazquez P, Bashan Y., The role of sediment microorganisms in the productivity, conservation, and rehabilitation of mangrove ecosystems: an overview. Biol Fert Soils, 2001, 33:265-278.

[3] Sahoo K, Dhal NK., Potential microbial diversity in mangrove ecosystems: a review. Indian J Mar Sci, 2009, 38(2): 249-256.

[4] Sengupta A, Chaudhuri S., Ecology of heterotrophic denitrogen fixation in the rhizosphere of mangrove plant community at the Ganges river estuary in India. Oecologia, 1991, 87: 560564.

[5] Holguin G, Guzman MA, Bashan Y., Two new nitrogen-fixing bacteria from the rhizosphere of mangrove trees: their isolation, identification and in vitro interaction with rhizosphere Staphylococcus sp. FEMS Microbiol Ecol, 1992, 101: 207-216.

[6] Alongi DM, Christoffersen P, Tirendi F., The influence of forest type on microbial nutrient relationships in tropical mangrove sediments. J Exp Mar Biol Ecol, 1993, 171(2): 201-223.

[7] Vazquez P, Holguin G, Puente ME, Lopez-Cortes A, Bashan Y., Phosphate-solubilizing microorganisms associated with the rhizosphere of mangroves in semiarid coastal lagoon. Bio Fertil Soils, 2000, 30: 460-468.

[8] Andreote FD, Jiménez DJ, Chaves D, Dias ACF, Luvizotto DM, Dini-Andreote F, Fasanella CC, Lopez MV, Baena S, Taketani RG, Melo IS., The microbiome of Brazilian mangrove sediments as revealed by metagenomics. PLoS ONE, 2012, doi: 10.1371/journal.pone.0038600. 
221

222

223

224

225

226

227

228

229

230

231

232

[9] Xu Z, Hansen MA, Hansen LH, Jacquiod S, Sørensen SJ., Bioinformatic Approaches Reveal Metagenomic Characterization of Soil Microbial Community. PLoS ONE, 2014, 9(4): e93445. doi: 10.1371/journal.pone.0093445

[10] Paul EA., Soil Microbiology, Ecology and Biochemistry (Third Edition). Elsevier Inc., 2007, pp 389-430.

[11] Zhou J, Bruns MA, Tiedje JM., DNA recovery from soils of diverse composition. Appl Environ Microbiol, 1996, 62(2): 316-322.

[12] Hyatt D, LoCascio PF, Hauser LJ, Uberbacher EC., Gene and translation initiation site prediction in metagenomic sequences. Bioinformatics, 2012, 28(17): 2223-2230.

[13] Ye Y, Choi JH, Tang H., RAPSearch: a fast protein similarity search tool for short reads. BMC Bioinformatics, 2011, doi:10.1186/1471-2105-12-159.

[14] Zhao Y, Tang H, Ye Y., RAPSearch2: a fast and memory-efficient protein similarity search tool for next generation sequencing data. Bioinformatics, 2012, 28(1): 125-126.

[15] Huson DH, Mitra S, Weber N, Ruscheweyh HJ, Weber N, Schuster SC., Integrative analysis of environmental sequences using MEGAN4. Genome Res, 2011, 21: 1552-1560.

[16] Disz T, Akhter S, Cuevas D, Olson R, Overbeek R, Vonstein V Stevens R, Edwards RA., Accessing the SEED genome databases via web services API: tools for programmers. $B M C$ Bioinformatics, 2010, doi:10.1186/1471-2105-11-319.

[17] Bates ST, Berg-Lyons D, Caporaso JG, Walters WA, Knight R, Fierer N., Examining the global distribution of dominant archaeal populations in soil. ISME J, 2011, 5(5): 908-917.

[18] Hou W, Wang S, Dong H, Jiang H, Briggs BR, Peacock JP, Huang Q, Huang L, Wu G, Zhi X, Li W, Dodsworth JA, Hedlund BP, Zhang C, Hartnett HE, Dijkstra P, Hungate BA., A comprehensive census of microbial diversity in hot springs of Tengchong, Yunnan Province 
China using 16S RNA gene pyrosequencing. PLoS ONE, 2013, doi: 10.1371/journal.pone.0053350.

246 [19] Janssen PH., Identifying the dominant soil bacterial taxa in libraries of 16S rRNA and 16S rRNA genes. Appl Environ Microbiol, 2006, 72(3): 1719-1728.

248 [20] dos Santos HF, Cury JC, do Carmo FL, dos Santos AL, Tiedje J, van Elsas JD, Rosado AS, 249 Peixoto RS., Mangrove bacterial diversity and the impact of oil contamination revealed by pyrosequencing: bacterial proxies for oil pollution. PLoS ONE, 2011, doi: 10.1371/journal.pone.0016943

[21] Jeffries TC, Seymour JR, Gilbert JA, Dinsdale EA, Newton K, et al., Substrate Type Determines Metagenomic Profiles from Diverse Chemical Habitats. PLoS ONE, 2011, 6(9): e25173. doi: 10.1371/journal.pone.0025173

[22] Ghosh A, Dey N, Bera A, Tiwari A, Sathyaniranjan KB, Chakrabarti K, Chattopadhyay D., Culture independent molecular analysis of bacterial communities in the mangrove sediment of Sundarban, India. Saline Syst, 2010, 6: 1 doi: 10.1186/1746-1448-6-1. heterogeneity of Fe(III)-reducing microorganisms in coal mining-associated lake sediments. Appl Environ Microbiol, 2008, 74(4): 1019-1029. 


\begin{tabular}{|l|l|l|}
\hline Parameter & Unit & RA \\
\hline Arsenic (As) & $\mathrm{mg} / \mathrm{kg}$ & $\mathrm{ND}(<0.5)$ \\
\hline Cadmium $(\mathrm{Cd})$ & $\mathrm{mg} / \mathrm{kg}$ & 0.10 \\
\hline Lead $(\mathrm{Pb})$ & $\mathrm{mg} / \mathrm{kg}$ & 25.34 \\
\hline Mercury $(\mathrm{Hg})$ & $\mathrm{mg} / \mathrm{kg}$ & $\mathrm{ND}(<0.05)$ \\
\hline $\mathrm{pH}(10 \% \mathrm{w} / \mathrm{w})$ & - & 5.1 \\
\hline Phosphorus & $\mathrm{mg} / \mathrm{kg}$ & 449.70 \\
\hline Sulfur & $\mathrm{mg} / \mathrm{kg}$ & 1374.52 \\
\hline Carbon:Nitrogen & $\%$ & $5.19: 0.31$ \\
\hline
\end{tabular}

264 Table 1. Results of biochemical analyses of the soil sample. ND: not detected. 


\begin{tabular}{|l|c|}
\hline Parameters & RA \\
\hline Total no. of sequences (bp) & 24227393584 \\
\hline Total no. of quality sequences (bp) & 20907568942 \\
\hline Average quality read length (bp) & \\
\hline Total no. of contigs ( $>400 \mathrm{bp})$ & 56.81 \\
\hline Total no. of contigs length (bp) & 541 \\
\hline Coverage & \\
\hline Percentage of hits against NCBI 16S & \\
\hline Microbial database (\%) & \\
\hline Total CDS assigned to SEED categories & \\
\hline
\end{tabular}

267 Table 2. Reads statistics. The numbers of reads were generated by Illumina HiSeq 2000. The

268 RA sample showed good quality of reads in term of number, length and number of contigs 269 generated. 
272 Figure 1 Bacteria and Archaea. The majority of the reads were assigned to the domain Bacteria

273 by MEGAN. The number of reads detected for archea was significantly higher than previously

274 reported.

275 Figure 2a The segregation of phyla in the domain Bacteria. Proteobacteria was the dominant 276 phylum in the samples with almost half of the reads assigned to this particular phylum.

277 Figure 2b Classes of bacteria. Classes of bacteria that were present in the RA sample at more 278 than $1 \%$.

279 Figure 2c Bacterial genera. The genera shown are those that had more than 1\% reads. Bacterial genera detected in RA sample in which Acidobacterium was the most abundant genus.

Figure 3 Classes of Proteobacteria. Deltaproteobacteria was the most abundant class in RA samples.

Figure 4 Order level of Deltaproteobacteria. Syntrophobacterales were the most abundant order.

Figure 5 The most abundant genera in Syntrophobacterales. Syntrophobacter was the most abundant genus. of Crenarchaeota.

Figure 7 Gene ontology. The SEED classification by MEGAN. 


\section{Percentage of assigned reads to domain}
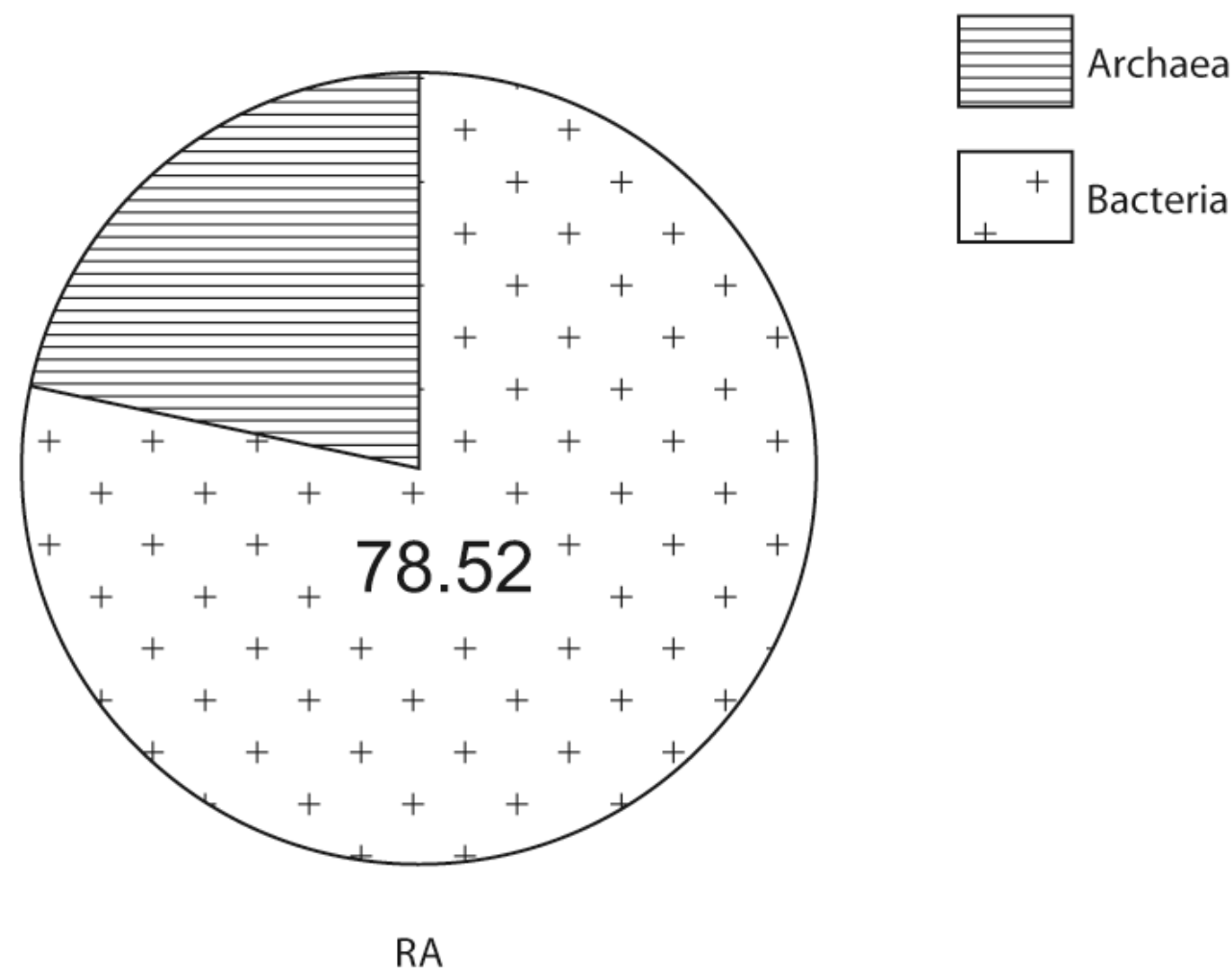

Figure 1 

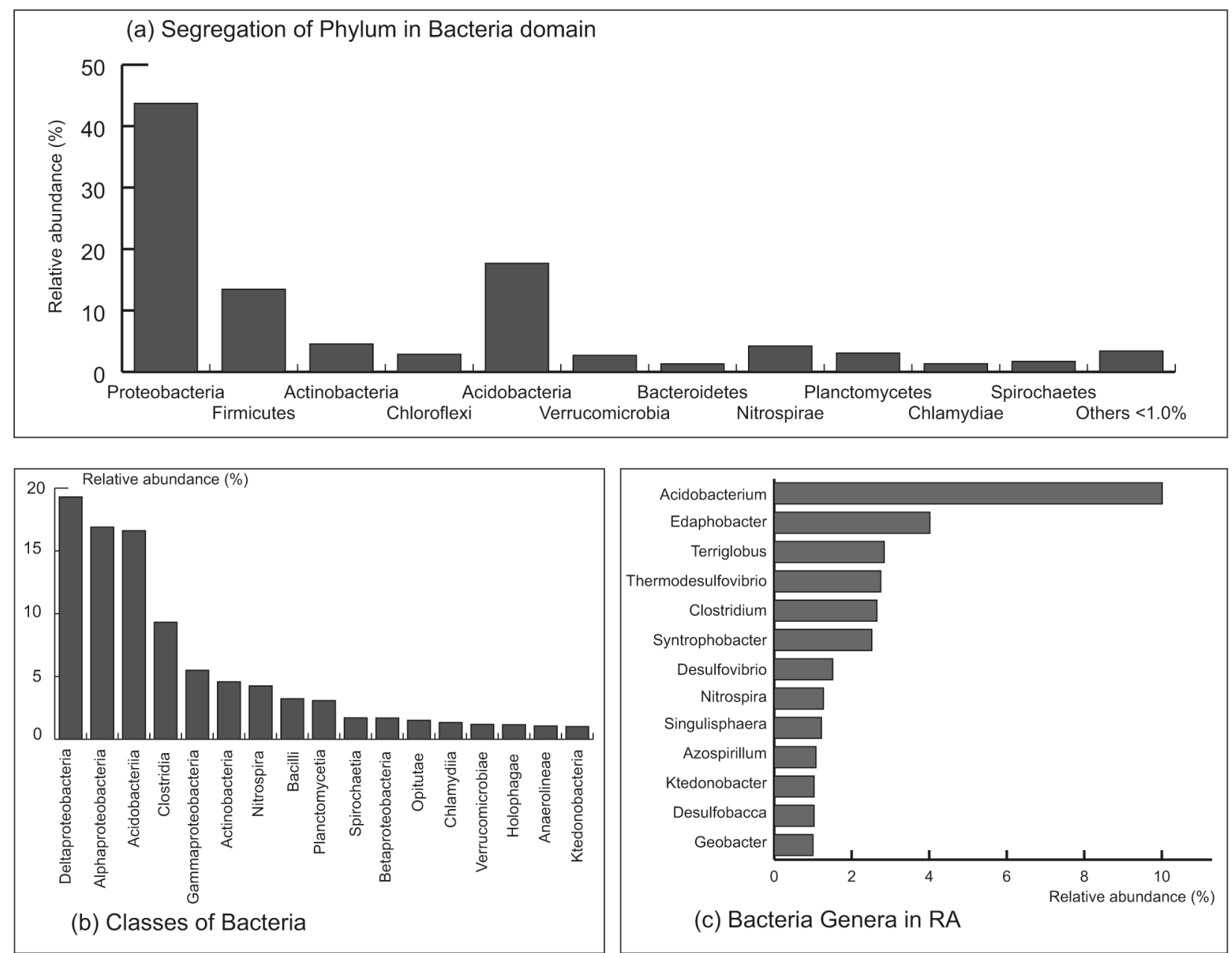

Figure 2 


\section{Classes of Photobacteria}

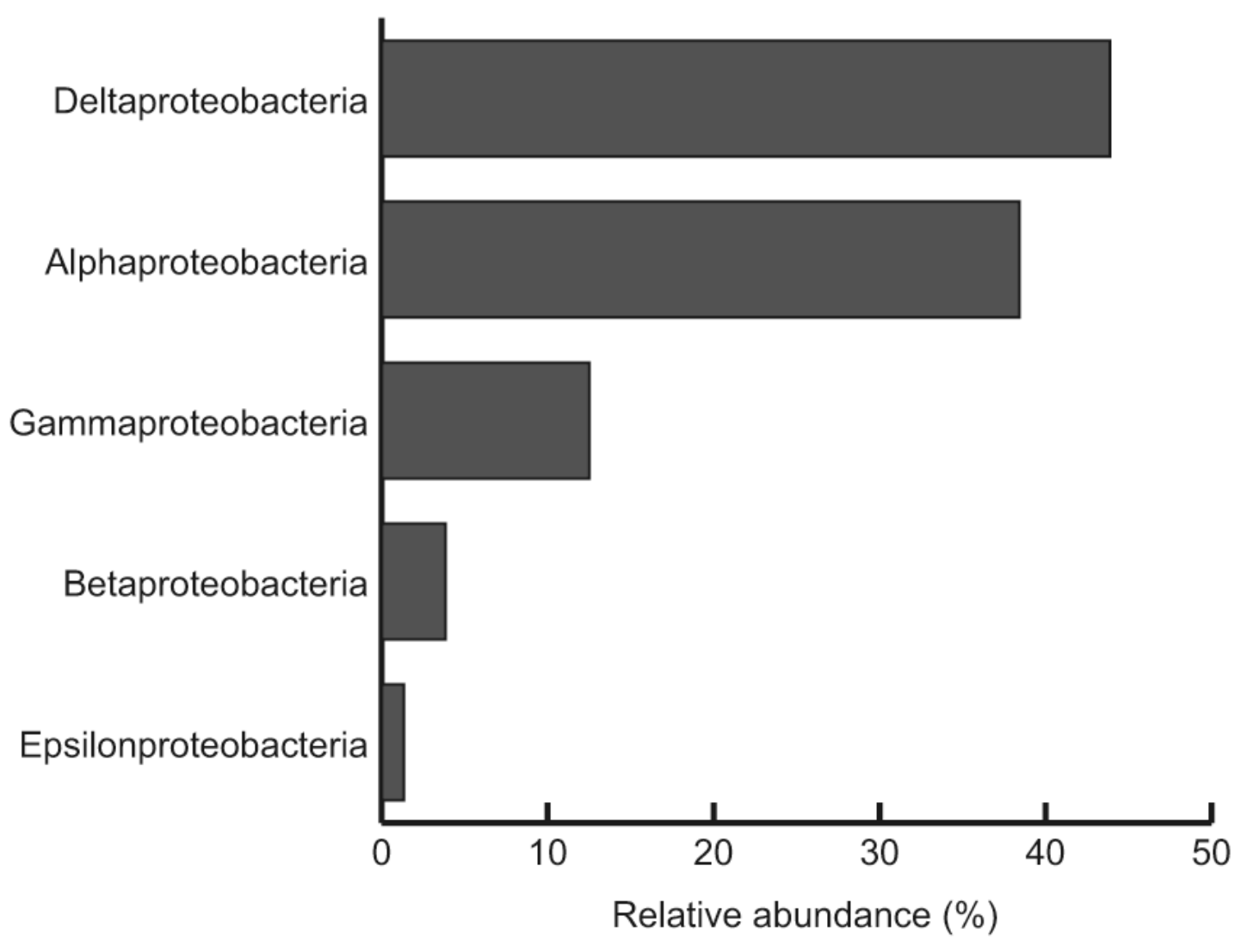

$299 \quad$ Figure 3 


\section{Order level of Deltaprotobacteria}

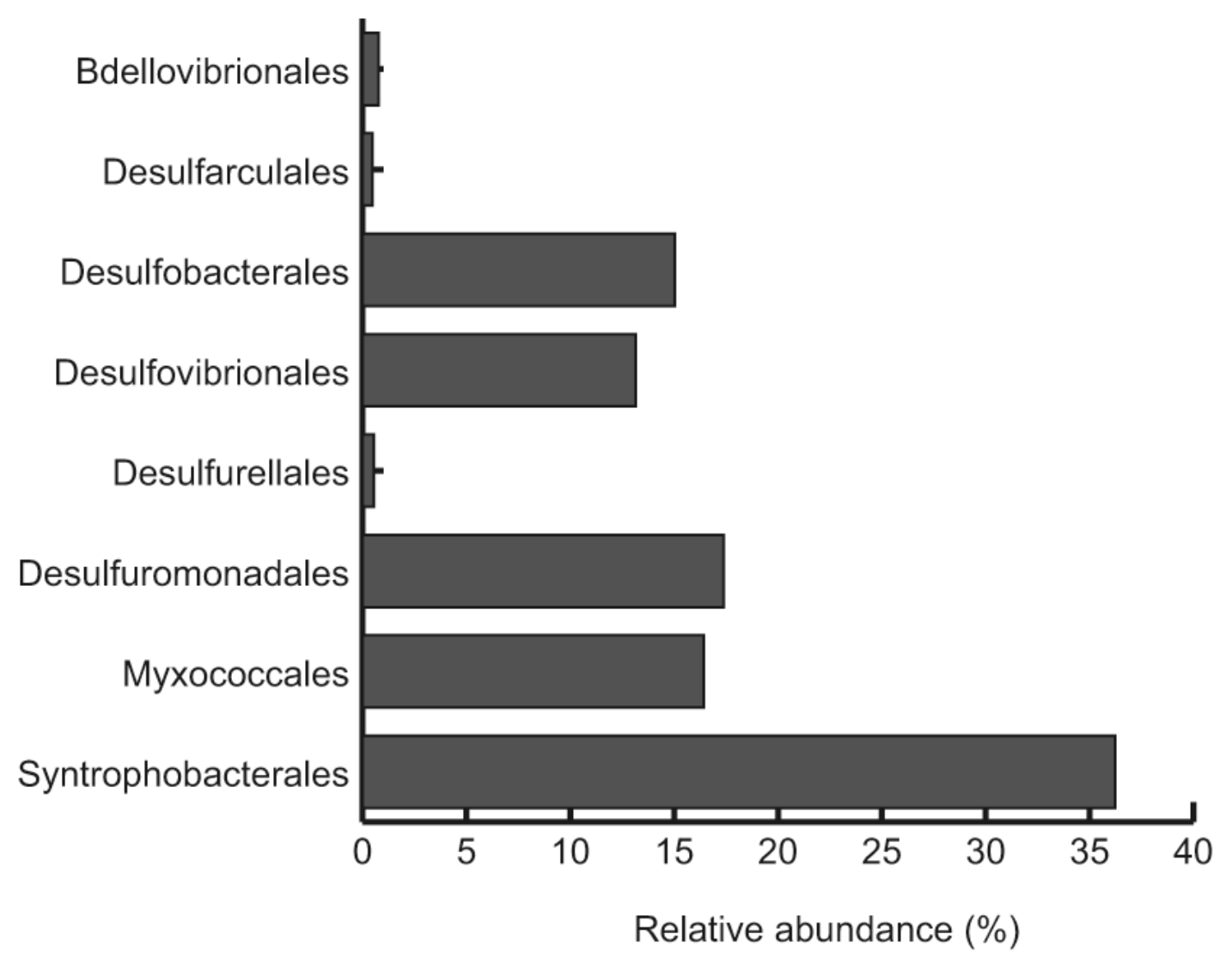




\section{Genus level of Syntrophobacterales}

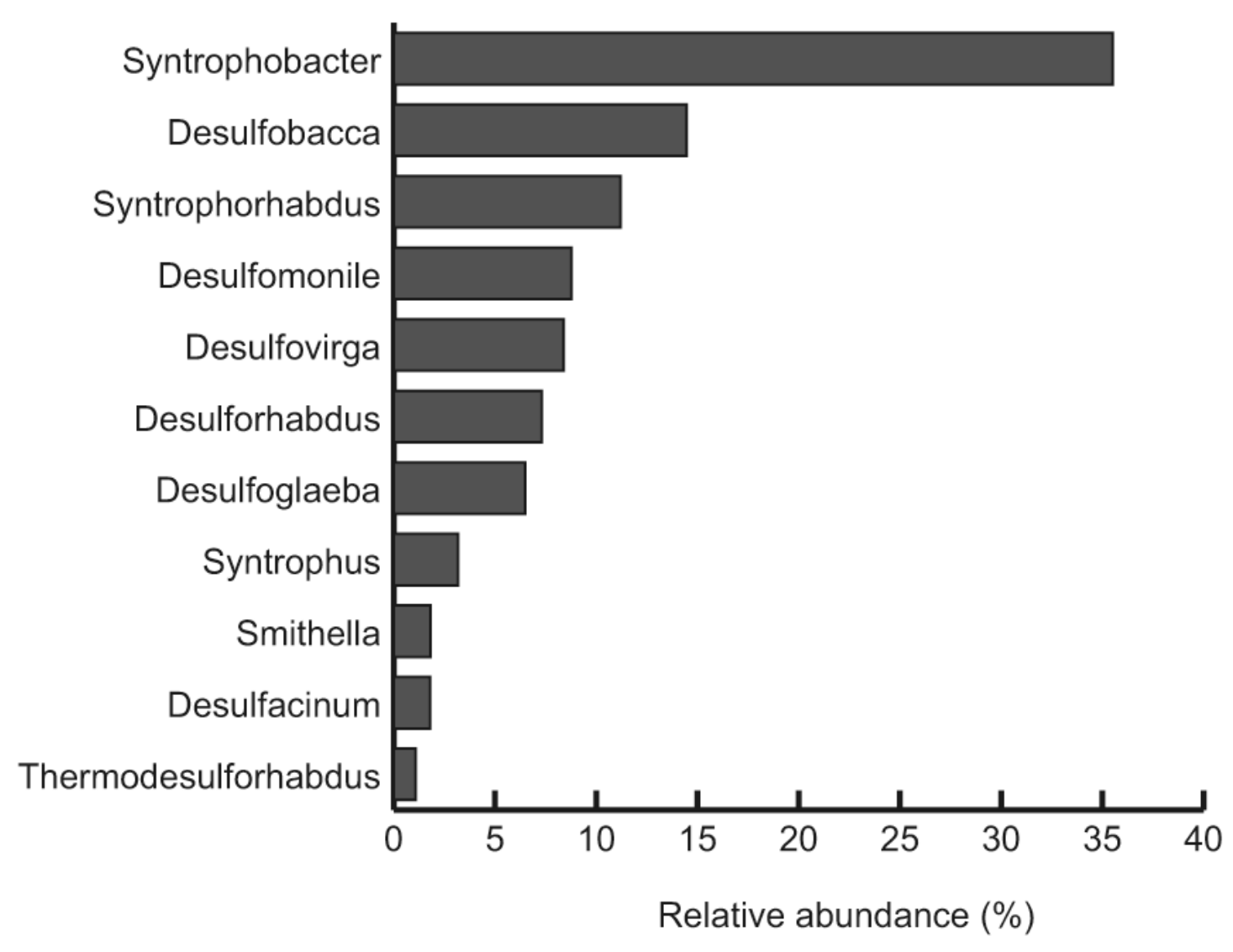

$305 \quad$ Figure 5 


\section{Percentage Distribution of Archaea Phylum}
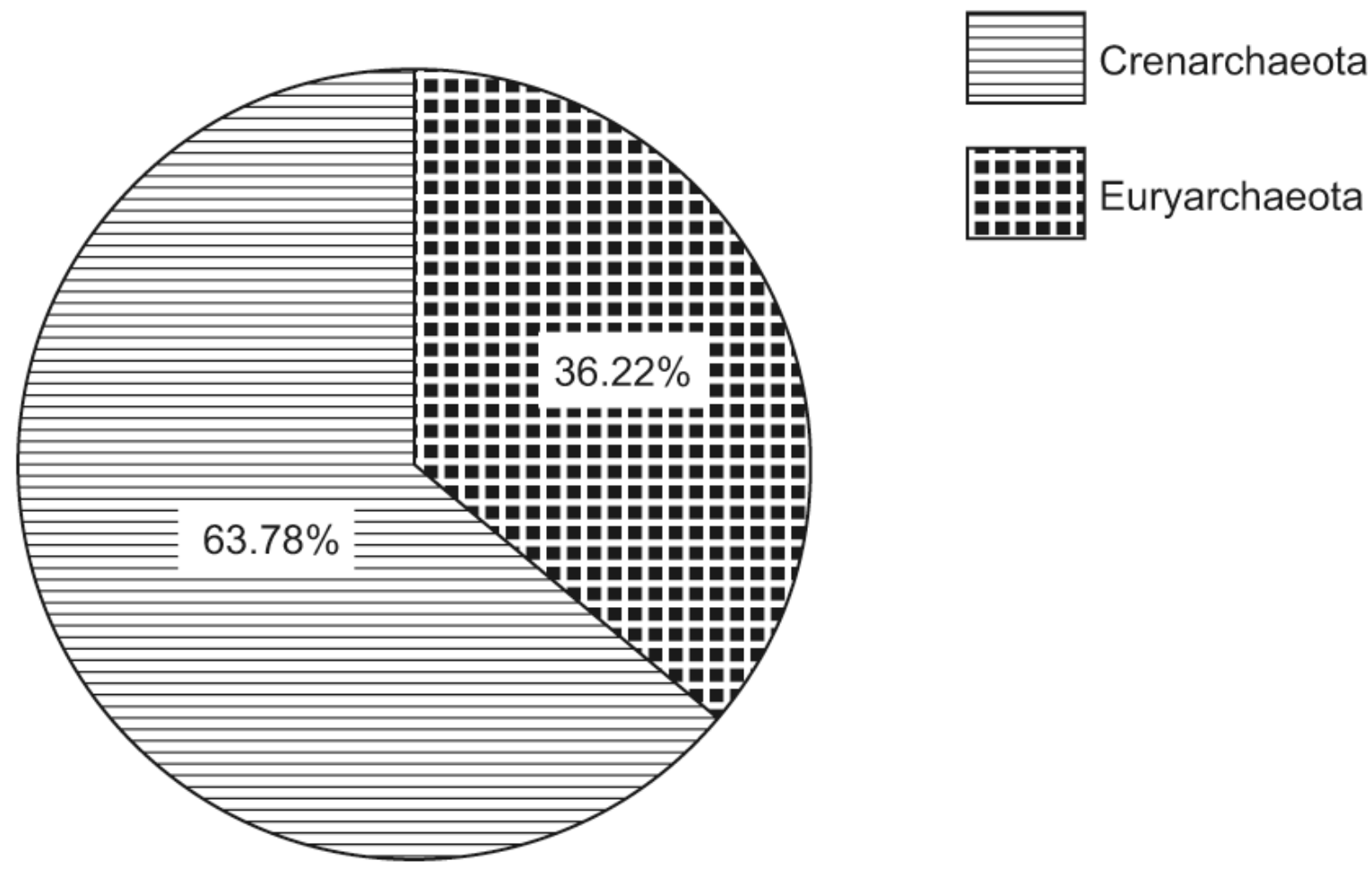

鰛 Euryarchaeota

Figure 6

309 
bioRxiv preprint doi: https://doi.org/10.1101/018895; this version posted May 3, 2015. The copyright holder for this preprint (which was not certified by peer review) is the author/funder. All rights reserved. No reuse allowed without permission.

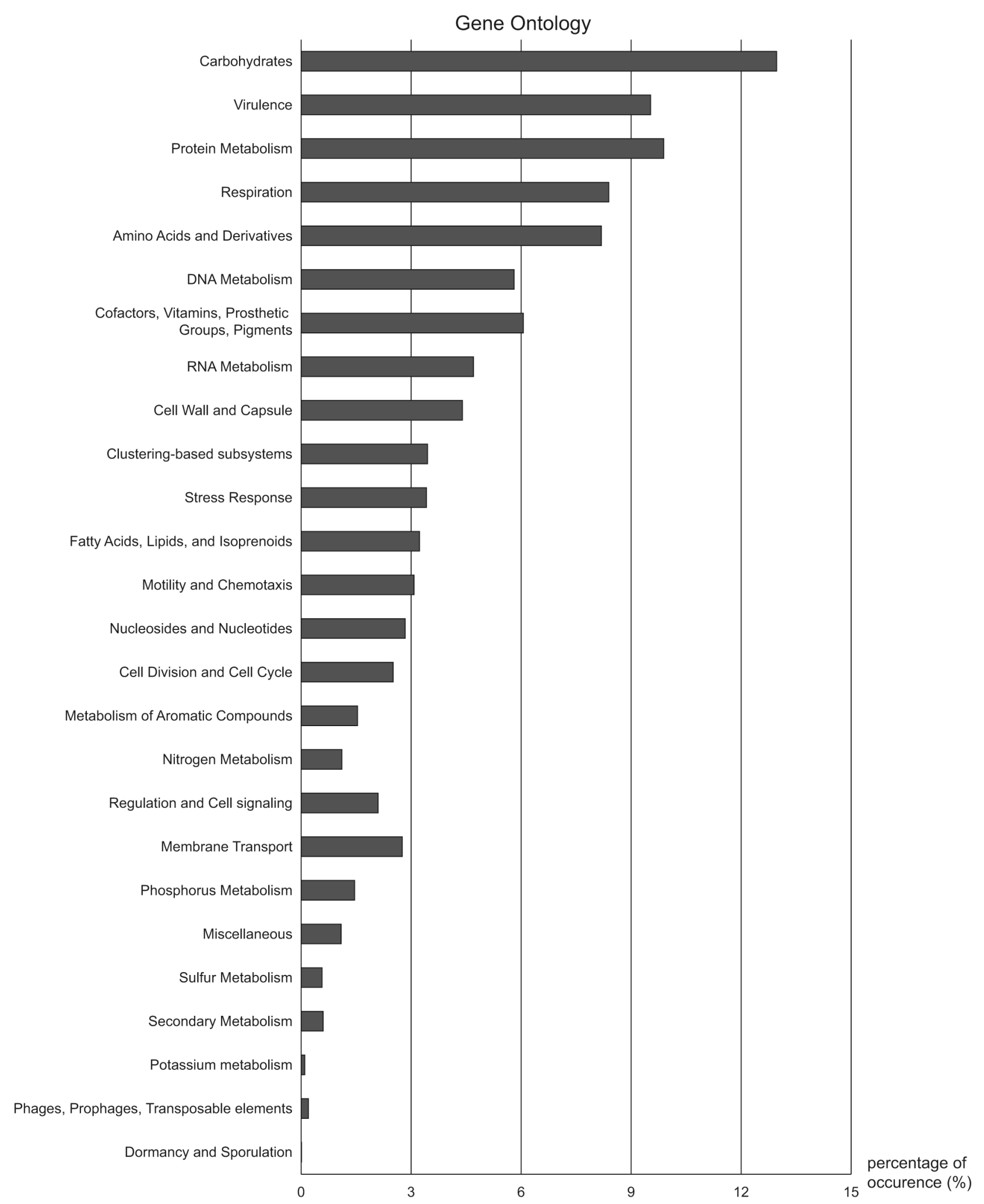

$311 \quad$ Figure 7 